\title{
Salmonella Typhi: from a Human Pathogen to a Vaccine Vector
}

\author{
Xiao-Lian Zhang ${ }^{1,2}$, Victor Tunje Jeza ${ }^{1}$ and Qin Pan $^{1}$
}

Salmonella (S.) typhi is an important intracellular pathogen. Among the more than 2,300 closely-related Salmonella serovars bacteria recognized, $S$. typhi is the only one that is pathogenic exclusively for humans, in whom it causes typhoid or enteric fever. The pathogen has been around for many years and many studies have been done in an effort to combat it. Molecular and biologic features of $S$. typhi and host factors and immune responses involved in Salmonella invasion have been extensively studies. Vaccines that have been developed most notably are Vi polysaccharide and Ty21a. However, as the results show, there is still a long way to go. It is also shown that multi-drug resistance has occurred to the few available antibiotics. More and more studies have shown that Salmonella can be used as a vaccine vector carrying antigens of other pathogens. This has been promising in that the immune system can be elicited in response to both the Salmonella bacteria and the antigen of the pathogen in question. This review aims to highlight some of the milestones attained in the fight against the disease from the time $S$. typhi was seen as a pathogen causing typhoid fever to the use of Salmonella as a vaccine vector. Cellular \& Molecular Immunology. 2008;5(2):91-97.

Key Words: Salmonella typhi, vaccine vector, typhoid, Vi polysaccharide, Ty21a

\section{Introduction}

Salmonella is the causative agent of salmonellosis. It is a rod-shaped gram-negative facultative anaerobe bacterium belonging to the Enterobacteriaceae family. Among more than 2,300 closely-related Salmonella serovars recognized, $S$. Typhi and Paratyphi are pathogenic exclusively for humans, and cause systemic infections and typhoid fever, whereas others such as S. Typhimurium cause gastroenteritis (1).

Salmonellosis is more prevalent in developing parts of the world in Africa, Asia, and South America. South Asia are at highest risk for infections that are nalidixic acid-resistant or multidrug-resistant (i.e., resistant to ampicillin, chloramphenicol, and trimethoprim-sulfamethoxazole). In humans, salmonellosis is seen in two kinds of viz. enteric fever which can be typhoid or paratyphoid and gastroenteritis which is non-typhoidal. Typhoid fever is an acute, life-threatening febrile illness caused by the bacterium S. Typhi and

\footnotetext{
${ }^{1}$ Department of Immunology, The State Key Laboratory of Virology, Hubei Province Key Laboratory of Allergy and Immune-related Diseases, Wuhan University, School of Medicine, Wuhan 430071, China;

${ }^{2}$ Corresponding to: Dr. Xiao-Lian Zhang, Department of Immunology, Wuhan University, School of Medicine, 165 Donghu Road, Wuhan, 430071, P.R. China. Tel: +86-27-8733-1183, Fax: +86-27-8733-6380, E-mail: ZhangXL65@whu.edu.cn
}

Received Nov 25, 2007. Accepted Feb 24, 2008.

Copyright (C) 2008 by The Chinese Society of Immunology
Paratyphi, and there are estimated 20 million cases and 200,000 deaths worldwide each year (2).

There have been vaccines to salmonellosis as well. However, the immune system has not been able to mount a lasting immune response to Salmonella infections and the reason for this still remains elusive. This has called for extensive studies on new therapies. Indeed, new vaccines have been developed. Further, it has been found that a highly immunogenic live oral Salmonella vaccine would ideally be suited as a carrier of genes that express protective antigens cloned from other antigens (3-5) and such hybrid recombinant Salmonella vaccines are expected to invoke protective immunity against both the carrier strains as well as the foreign antigens (6). This is the basis of Salmonella vaccine vector and this review seeks to illustrate this milestone in the scientific research done to date.

We begin by highlighting the molecular and biologic features of Salmonella, host immune responses of $S$. typhi infections, therapeutic measures to salmonellosis and the challenges associated with them, and lastly but not least, the use of Salmonella typhi as a vaccine vector.

\section{Molecular and biologic features of Salmonella typhi}

The genome of $S$. typhi is approximately 5 million base pairs long and codes for some 4,000 genes of which more than 200 are functionally inactive. This is in line with other genomes of enteric bacteria sequenced so far which feature a single chromosome with 4.3-5.0 $\mathrm{Mb}$ in length (7-10). A comparison 


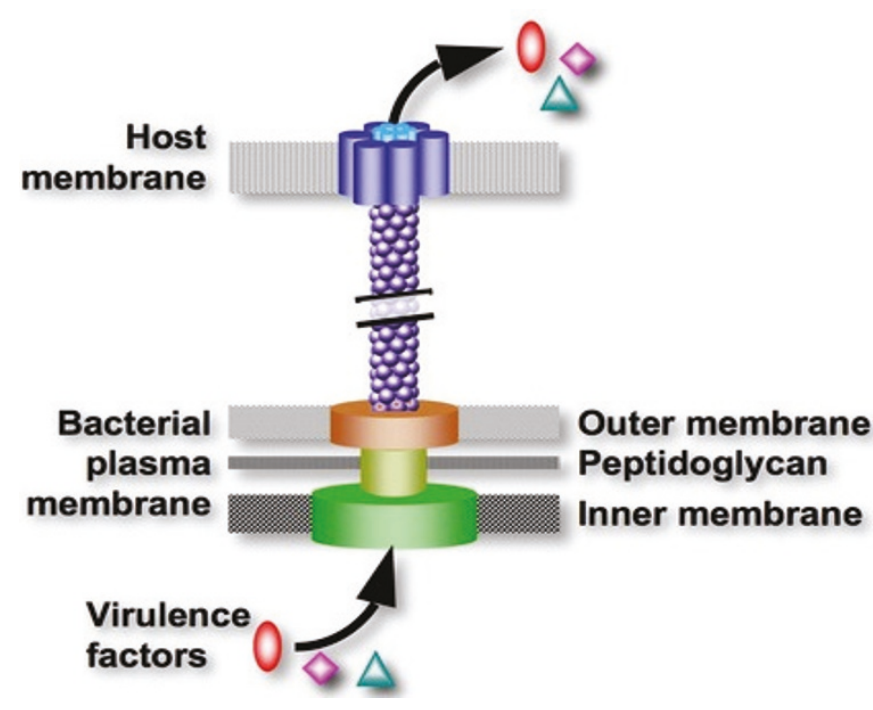

Figure 1. The TTSS is used by the bacteria to translocate virulence factors to the host during infections. Without it, invasion to host cells can not take place.

of $S$. typhi isolates from around the world indicate that they are highly related and that they emerged from a single point of origin approximately 30,000 to 50,000 years ago (11). Different strains may also harbor extrachromosomal DNA in the form of plasmids which usually carry virulence or antibiotic resistance genes (11).

The genes for virulence factors cluster in pathogenicity islands (PI) after a foreign genome is integrated into the bacterial chromosome. Non-pathogenic related species of Salmonella do not have PIs. PI gene expression is generally limited to specific host compartments. They often "plug" in to endogenous two-component housekeeping regulators to "sense" where they are within a host and regulate expression accordingly (12). Two major PIs encode type three secretion system (TTSS) that translocates bacterial virulence proteins into host cells during infections (12) (Figure 1).

The TTSS and its effectors encoded by Salmonella pathogenesis island 1 (SPI-1) are required for invasion of epithelial cells (12) and is activated under conditions thought to be present in the intestinal lumen before host cell invasion (13). The SPI-1 secreted effectors SopE and SopE2 act as guanine-nucleotide-exchange-factors (GEFs) for the small GTPases Cdc42 and Rac (14) (Figure 2). Additional SPI-1translocated effectors of Salmonella affect actin dynamics during the invasion process. SipA binds and stabilizes actin and SipC, which forms part of the TTSS delivery pore, nucleates and bundles actin while anchored in the host cell membrane (15). Salmonella also alters the actin cytoskeleton, through manipulation of phosphoinositides. The plasma membrane is intimately associated with the actin cytoskeleton, and this interaction depends on phosphatidylinositol 4,5-bisphosphate (PtdIns $\left.(4,5) \mathrm{P}_{2}\right)(16)$. SigD/SopB is an SPI-1 translocated inositol phosphatase that induces the rapid disappearance of $\operatorname{PtdIns}(4,5) \mathrm{P}_{2}$ from invaginating regions of

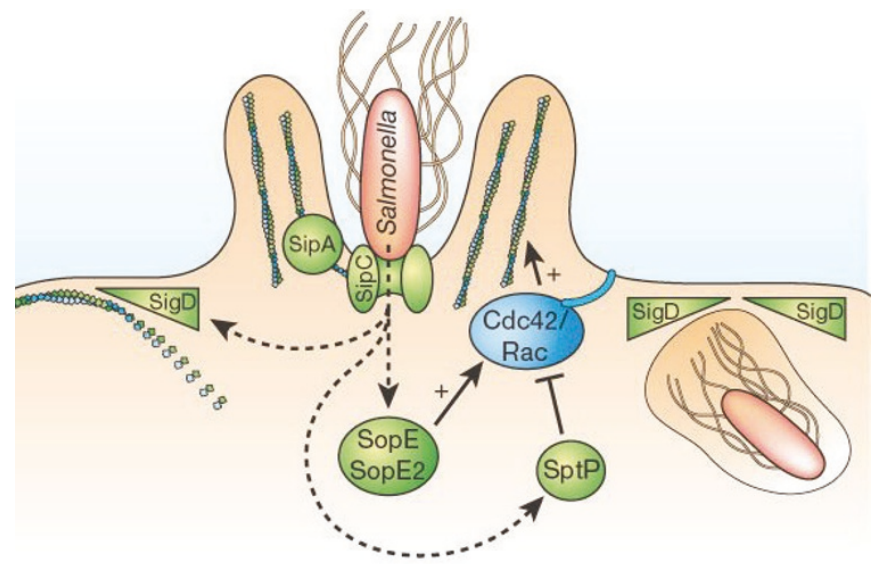

Figure 2. Entry into host cells is mediated by the TTSS and its effectors encoded by SPI-1. Membrane attachment to the cortical actin cytoskeleton is loosened by $\mathrm{SigD} / \mathrm{SopB}$. SopE and SopE2 enhance $\mathrm{Cdc} 42$ and Rac1 activity directly by acting as guaninenucleotide-exchange factors. SipA and SipC alter cytoskeletal structure, SipC by nucleating actin and initiating polymerization and SipA by binding actin and modulating actin bundling. These cytoskeletal rearrangements are downregulated by the GAP (GTPase-activating protein) activity of SptP, which inactivates Cdc42 and Rac. SigD also is involved in sealing invaginating regions of the plasma membrane to form intracellular vacuoles.

the membrane during Salmonella invasion. This increases elasticity to facilitate the remodelling of the plasma membrane associated with Salmonella entry (16). PtdIns $(4,5) \mathrm{P}_{2}$ has also been implicated in vesicle fission during the creation of phagosomes and clathrin-coated vesicles, and accordingly, SigD also is involved in sealing plasma membrane invaginations to form bona fide vacuoles (17). After invasion, an additional SPI-1 effector, SptP, acts as a GTPase-activating protein (GAP) for $\mathrm{Cdc} 42$ and Rac1, thereby inactivating these $\mathrm{G}$ proteins and returning cell morphology to a relatively normal state (17). SptP is a bifunctional protein, with its GAP domain at the amino terminus, and a protein tyrosine phosphatase domain at the carboxy terminus (18). A potential target for the tyrosine phosphatase activity of SptP is the intermediate filament protein vimentin, which is recruited to the membrane ruffles stimulated by Salmonella (18). Other studies have also identified another intermediate filament protein involved in Salmonella entry, SipC, which binds cytokeratins and expression of dominant negative cytokeratin-18 inhibits Salmonella entry into HEp2 cells (19) (Figure 2). Salmonella pathogenesis island 2 (SPI-2) encodes a second TTSS, effector proteins, molecular chaperones, and a two component regulatory system that activates SPI-2 promoters (14). This pathogenicity island and related effectors are required for intracellular survival and replication at systemic sites of infection. Furthermore, studies by Coombes and others have shown that negative regulation of SPI-2 is required for full typhoid pathogenesis and they (Coombes and others) have also identified a protein in Salmonella 


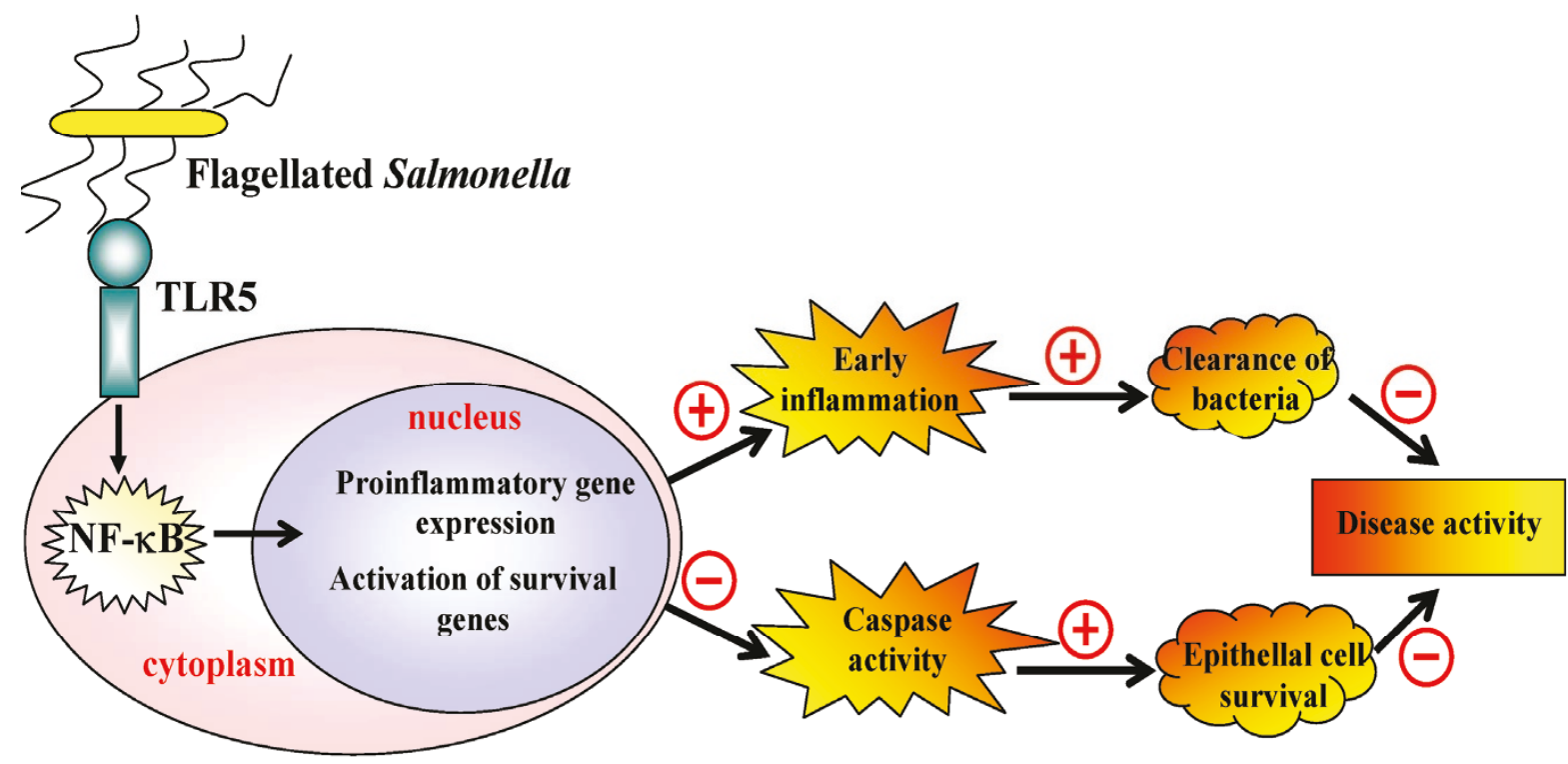

Figure 3. Flagellin of Salmonella bacteria stimulates the activations of TLR5 and NF-אB, suppresses epithelial apoptosis and limits disease during enteric infection.

enterica called $\mathrm{Ydg} \mathrm{T}$ that exerts a negative regulatory activity on SPI-2 (19).

Other than these two major PIs, there are other PIs that have been identified most of which are of utmost importance to the virulence and survival of the bacterium. Such islands are SPI-3, SPI-4 and SPI-5. It has been suggested that when genes associated with the SPI-3, SPI-4, and SPI-5 together with SPI-1 and SPI-2 are inactivated, S. typhi loses the ability to express several virulence-associated traits, a factor that could begin to explain the loss of host range of these serovars. Another important island is the SPI-7 which is responsible for the production of the $\mathrm{Vi}$ polysaccharide capsule (20). This island is $134 \mathrm{~kb}$ in length and encodes a variety of putative virulence-associated gene clusters, including the Vi locus, a phage encoding the SopE effector protein of PSI-1 (21-23), a type IV pilus, and a putative type IV secretion system (24-27). The type IV pilus is involved in aiding attachment to eukaryotic cells (24-27) and the SopE prophage harbors a gene encoding an effector protein secreted through the TTSS (14).

In addition, SPI-7 has a viaB operon which encodes the gene responsible for the synthesis and transport of the virulence antigen Vi $(28,29)$. Studies have shown that Vi antigen may be important in the survival of the bacterium within macrophages though this may not be in cellular invasion of the macrophage or the intestinal wall $(14,30)$. Unlike most other serovars, Salmonella typhi expresses the Vi polysaccharide capsule, an important virulent factor. However, its presence only increases the infectivity of Salmonella typhi and the severity of the disease but is not essential for infection since it has been shown that Vi negative mutants are able to cause a typhoid-like illness in human volunteers $(7,31)$. Supporting this view is the fact that there have been reports of outbreaks of typhoid fever caused by Vi-negative Salmonella typhi (32).

\section{Host factors and immune responses involved in Salmonella invasion}

Salmonella infections are usually acquired by ingestion of contaminated food or water. Once ingested, the organisms multiply in the small intestine over the period of 1-3 weeks, breech the intestinal wall, and spread to other organ systems and tissues.

Salmonella interactions with nonphagocytic cells and phagocytic cells. The primary site of invasion of Salmonella strains is thought to be the M cells in the Peyer's patches. Starting in the late 1990s, Salmonella flagella was highlighted in host early innate immunity against Salmonella beacause the flagella was identified by several independent groups as the mediator that causes intestinal epithelial or macrophage inflammation following infection. CiacciWoolwine et al. and Wyant et al. reported that Salmonella enterica serovar Typhi flagella induced cytokine release from human monocytes and impaired antigen presentation by human macrophages (33-35). Flagellin of Salmonella suppresses epithelial apoptosis and limits disease during enteric infection (Figure 3) (36). The molecular basis of these effects was revealed by Hiyashi et al., who demonstrated that flagellin was the component that activated TLR5, and subsequent work in many laboratories confirmed this finding for flagellins from various organisms $(37,38)$. Moreover, recent studies have identified Ipaf as an essential sensor for cytoplasmic flagellin $(39,40)$. Feuillet et al. generated TLR5-deficient mice and showed that TLR5 was crucial for 
the in vivo recognition of flagellin but also may participate in the detection of systemic infection by $S$. typhimurium (41). After engagement, TLR5 activates NF- $\mathrm{KB}$ and MAPKs, leading to the secretion of many cytokines, including IL-6, IL-12, and TNF- $\alpha$, whereas Ipaf permits the activation of caspase- 1 and secretion of mature IL- $1 \beta$.

It has been demonstrated that the activation of macrophages by LPS from Salmonella species also results in the release of a variety of inflammatory cytokines, such as IL-6 and IFN- $\beta$ (42), which were not detected in macrophages of TLR4 knockout mice (43). After binding to LPS, in association with the proteins MD2 and CD14, TLR4 dimerizes and undergoes a conformational change required for the recruitment of downstream Toll/interleukin-1 receptor (TIR) domain-containing adaptor molecules to activate both $\mathrm{NF}-\mathrm{\kappa B}$ and the mitogen-activated protein kinases (MAPKs) (44). Other studies have shown that TLR4 triggers the early response to Salmonella and that TLR4 and TLR2 are required sequentially for efficient macrophage function in Salmonella infections (45). TLR2 can recognize Salmonella bacterial lipoproteins and lipoteichoic acid $(46,47)$, probably in cooperation with TLR6 and/or TLR1 (48-50). TLR9 is activated by bacterial DNA (detecting unmethylated $\mathrm{CpG}$ motifs) (51). Totemeyer et al. have demonstrated that TLR1, TLR2, and TLR9 are up-regulated while TLR6 is downregulated which accounts for the plateau phase observed during sublethal Salmonella enterica serovar Typhimurium infection (51). Their results suggest that in addition to TLR4, the TLR2-TLR1 complex and TLR9 may play a role in controlling infection, particularly in the later stages when the bacterial growth is suppressed, possibly at the adaptive phase of the immune response (51).

The phase of early innate immunity is followed by activation of a complex host response that suppresses the growth of bacteria in tissues. Both macrophages and $\mathrm{T}$ cells may be involved in cell-mediated immunity to Salmonella infection, while antibody also plays a role. Central to the pathophysiology of all human salmonellosis is the induction of a strong host innate immune/inflammatory response (52). Both the host and pathogen have evolved mechanisms of triggering host responses. Induction of antimicrobial pathways during early-phase immune response to Salmonella spp. in murine macrophages: gamma interferon (IFN- $\gamma$ ) and macrophage migration inhibitory factor (MIF) play pivotal roles in immunity against Salmonella bacteria (53). Upregulation of IFN- $\gamma$ receptor $\alpha$ expression is required for NADPH phagocytic oxidase gp91-stimulated oxidative burst and control of virulent Salmonella spp. (54).

\section{Therapeutic measures to Salmonella typhi infections and the challenges associated with them}

Vaccination is one of the most important therapeutic means directed towards important antigens encoded by the bacterium. It can provide a near-term solution, as demonstrated in Thailand, where mass vaccination of school children with injectable inactivated, whole-cell vaccines in the 1970s and 1980s led to a sharp decrease in the incidence of typhoid fever which is credited for largely controlling the disease. However, because of their high rates of side effects, these older generation vaccines have generally been abandoned as public health tools (55). Fortunately, newer generation typhoid vaccines that have been available for approximately two decades have proved to be extremely safe. These are Vi polysaccharide and Ty21a vaccines (55). Both parental and live oral vaccines of $\mathrm{Vi}$ polysaccharide and Ty21a are clinically available for typhoid fever although there are some problems related to side effects and efficacy.

The development of a safe but highly immunogenic live oral typhoid vaccine against typhoid fever will have advantages over the currently available reactogenic whole-cell parenteral typhoid vaccines and the well tolerated but modestly immunogenic live oral vaccine strain Ty21a. However, development of these live oral serovar Typhi vaccines has been difficult, since little information is available regarding the mechanisms behind protective immunity and immunological memory for serovar Typhi or the interaction between the bacterium and the gut microenvironment. Another obstacle in the development of these live oral vaccines is the variety of typhoidal and nontyphoidal salmonelloses caused by various serovars and strains. Currently, the successes of clinical trials performed on the $\triangle a r o C / \triangle s s a V$ mutant to individuals in developing countries is a significant challenge. The new trials testing this vaccine strain in adults and children from developing countries are under way, and if successful, it could be the first single-dose oral vaccine for typhoid fever available worldwide (56).

Two vaccine strains harboring deletion mutations in aroC and $\operatorname{aroD}$ have been evaluated as candidate live oral vaccines in adult volunteers (57). One of these strains, CVD 908, a derivative of the wild-type strain Salmonella typhi Ty2, was well tolerated and highly immunogenic when given to volunteers in phase 1 studies. Another $\triangle$ aroC/ $\triangle a r o D$ strain, CVD 906, a derivative of wild-type strain ISP1820, was highly immunogenic but caused fever in a significant proportion of vaccines. Additional attenuating mutations in CVD 908 and CVD 906 were sought, a htrA gene locus, the resulting mutant is less virulent because of impaired ability to survive and/or replicate in host tissues (57).

Another Typhi vaccine candidate Ty800, is a derivative of $S$. typhi Ty2 and has a defined deletion mutation of the two-component virulence-regulating genes, phoP/phoQ. In human trials, Ty800 induced high-level immune responses with a single dose and without adverse side effects, except for diarrhea in a small number of individuals (58). Phase I/II clinical trials were undertaken by AVANT Immunotherapeutics, Inc. which aimed at determining the safety and immunogenicity of the single-dose, oral Ty800 vaccine.

Antibiotics directed towards Salmonella have also been recorded as success stories in fighting typhoid fever. The first antibiotic used to treat typhoid was chloramphenicol. This has been met with resistance by the bacterium and more so in developing countries where chloramphenical was the most affordable drug to use for enteric fevers (59). Newer 
antibiotics have been produced, an example of which is fluoroquinolones, which are better at treating the disease. But as the Salmonella bacterium continues to evolve, attaining new genes from the environments in forms of plasmids, prophages, and transposons via natural transformation, transduction and conjugation, these newer drugs will inevitablly be resisted sooner or later. Antibiotic resistance is notorious in developing countries where majority of the population is unable to afford full doses and in cases where people can afford, it may be the only drug affordable. Over use is also another factor contributing to drug resistance and this is seen in developed countries as well.

\section{Salmonella: a vaccine vector}

Typhoid fever affects millions of people worldwide and results in hundreds of thousands of death each year. This is to a large extent due to an increase in multi-drug resistance of Salmonella typhi. Consequently, the WHO has given a high priority to the improvement of vaccines against Salmonella typhi in an effort to attain greater control over the disease (60). The development of a safe but highly immunogenic live oral typhoid vaccine for use in such programs will have advantages over the currently available reactogenic wholecell parenteral typhoid vaccines and the well tolerated but modestly immunogenic live oral vaccine strain Ty21a (31, 61, 62). Salmonella typhi penetrates the gastrointestinal epithelial barrier and infects phagocytes mainly macrophages within the lamina propia where they adapt to prolonged survival thereby giving the bacteria an opportunity to spread to other systemic organs. Because of the natural tendency of the Salmonella typhi pathogen to spread to systemic tissues and persist in macrophages, a hybrid Salmonella typhi vaccine strain could be developed to carry other antigens as well which would be directed at developing immunity to other diseases. Salmonella vaccines are also capable of stimulating other T-cell subsets such as Th-2, cytotoxic, and memory $\mathrm{T}$ cells.

Several attenuated Salmonella typhi oral vaccines have been constructed to serve as vectors for heterologous antigens, including but not limited to antigens of Shigella sonnei, Escherichia coli, Vibrio cholerae, Bordetella pertussis, Plasmodium falciparum, B. anthracis, Helicobacter pylori, and Listeria monocytogens (63-68). As a vaccine against typhoid fever, Salmonella typhi Ty21a is a widely used licensed live vaccine that is exceedingly safe but requires three to four immunizations to induce long-term (at least 6-7 years) protective immunity in two thirds of immunized individuals (69). More highly immunogenic live S. typhi vaccines, such as CVD908-htrA (59), are currently being clinically evaluated for the safety.

Strains of S. Typhimurium and S. Typhi were first used as recombinant vectors for antigen delivery (70). Compared to injectable vaccines, oral delivery of attenuated Salmonellabased vaccines should result in increased compliance, safety, and ease of administration. Eliciting mucosal, cellular, and humoral immunity is desirable for protection against many of the organisms that infect at mucosal surfaces, including gastrointestinal, genital, and respiratory pathogens. Salmonellae are the most commonly studied vectors, perhaps in part because of the ease with which they are genetically manipulated, the existence of the serovar Typhimurium mouse model for preclinical work, and favorable prior human experience with the live attenuated vaccine U.S. Food and Drug Administration approved vaccine for typhoid fever, Ty21a. Ty21a serves as an important safety benchmark for researchers undertaking clinical trials. However, there are other safety considerations. The foreign genes inserted into the chromosome or foreign plasmid vectors carried by Salmonella-based vaccines should possess some containment features to minimize the possibility of transfer to and maintenance in other bacterial species. The plasmid vectors should therefore be nonconjugative, preferably be nonmobilizable, possess a narrow replicon host range, and not specify resistance to any antibiotic (71).

Recently a type IV pilus operon promoter controlling nucleocapsid gene expression of severe acute respiratory syndrome associated coronavirus (SARS-CoV) in $S$. typhi elicits full immune response by intranasal vaccination has been reported (72). This provides insights that type IV pilus operon promoter controlling SARS-CoV viral gene expression in $S$. typhi might be an attractive live vector vaccine against both infections of SRAS-CoV and $S$. typhi for it could induce mucosal, humoral, and cellular immune responses. However, further clinical studies are needed to assert this.

\section{Conclusion}

Salmonella typhi is a host specific pathogen affecting humans. Its virulence is aided by SPIs. SPI-1 is required for invasion of the bacteria to epithelial cells while SPI-2 is essential for survival and replication in the host cells. Salmonella has been around for many years and a lot of studies have been done in an effort to combat its infections. However, formidable challenges have been met in the process. Some of these challenges are multi-drug resistance of the bacteria and failure of vaccines to induce a lasting protective effect. Other challenges are just sheer lack of affordability of the means of treatment and preventive measures in the developing world.

Recently, Salmonella has emerged as a vaccine vector carrying antigens of other disease causing organisms, making it possible for a double protective therapy. However, further studies are needed to ascertain prophylactic use of these new modes of treatment to infectious diseases.

\section{Acknowledgements}

This work was supported by grants from National Natural Science Foundation of China (30670098, 30470087, and 30570070), the Major State Basic Research Development Program of China ("973" project 2005CB522901 and 
2006CB504300), and a grant from Hubei Province Science Technology Department (2006ABD007).

\section{References}

1. McClelland M, Sanderson KE, Spieth J, et al. Complete genome sequence of Salmonella enterica serovar Typhimurium LT2. Nature. 2001;413:852-856.

2. Crump JA, Luby SP, Mintz ED. The global burden of typhoid fever. Bull World Health Organ. 2004;82:346-353.

3. Aggarwal A, Kumar S, Jaffe R, et al. Oral Salmonella: malaria circumsporozoite recombinants induce specific $\mathrm{CD} 8^{+}$cytotoxic T cells. J Exp Med. 1990;172:1083-1090.

4. Formal SB, Baron LS, Kopecko DJ, et al. Construction of a potential bivalent vaccine strain: introduction of Shigella sonnei form I antigen genes into the galE Salmonella typhi Ty2Ia vaccine strain. Infect Immun. 1981;34:746-750.

5. Wu JY, Newton S, Judd A, et al. Expression of immunogenic epitopes of hepatitis B surface antigen with hybrid flagellin proteins by a vaccine strain of Salmonella. Proc Natl Acad Sci U S A. 1989;86:4726-4730.

6. Hone MD, Tacket CO, Harris AM, et al. Evaluation in volunteers of a candidate live oral attenuated Salmonella typhi vector vaccine. J Clin Invest. 1992;90:412-420.

7. McClelland M, Sanderson KE, Clifton SW, et al. Comparison of genome degradation in Paratyphi A and Typhi, humanrestricted serovars of Salmonella enterica that cause typhoid. Nat Genet. 2004;36:1268-1274.

8. Parkhill J, Dougan G, James KD, et al. Complete genome sequence of a multiple drug resistant Salmonella enterica serovar Typhi CT18. Nature. 2001;413:848-852.

9. Welch RA, Burland V, Plunkett G 3rd, et al. Extensive mosaic structure revealed by the complete genome sequence of uropathogenic Escherichia coli. Proc Natl Acad Sci U S A. 2002;99:17020-17024.

10. Yang F, Yang J, Zhang X, et al. Genome dynamics and diversity of Shigella species, the etiologic agents of bacillary dysentery. Nucleic Acids Res. 2005;33:6445-6458.

11. Baker S, Dougan G. The genome of Salmonella enterica serovar Typhi. Clin Infect Dis. 2007;45:S29-33.

12. Zhou D, Galán J. Salmonella entry into host cells: the work in concert of type III secreted effector proteins. Microbes Infect. 2001;3:1293-1298.

13. Baker S, Sarwar Y, Aziz H, et al. Detection of Vi-negative Salmonella enterica serovar Typhi in the peripheral blood of patients with typhoid fever in the faisalabad region of Pakistan. J Clin Microbiol. 2005;43:4418-4425.

14. Thomson N, Baker S, Pickard D, et al. The role of prophage-like elements in the diversity of Salmonella enterica serovars. J Mol Biol. 2004;339:279-300.

15. Fu Y, Galán JE. A Salmonella protein antagonizes Rac-1 and Cdc42 to mediate host-cell recovery after bacterial invasion. Nature. 1999;401:293-297.

16. Raucher D, Stauffer T, Chen W, et al. Phosphatidylinositol 4, 5-bisphosphate functions as a second messenger that regulates cytoskeleton-plasma membrane adhesion. Cell. 2000;100:221228.

17. Knodler LA, Steele-Mortimer O. Taking possession: Biogenesis of the Salmonella-containing vacuole. Traffic. 2003;4:587-599.

18. Murli S, Watson RO, Galán JE. Role of tyrosine kinases and the tyrosine phosphatase SptP in the interaction of Salmonella with host cells. Cell Microbiol. 2001;3:795-810.

19. Carlson SA, Omary MB, Jones BD. Identification of cytokeratins as accessory mediators of Salmonella entry into eukaryotic cells. Life Sci. 2002;70:1415-1426.

20. Hashimoto Y, Li N, Yokoyama H, Ezaki T. Complete nucleotide sequence and molecular characterization of $\mathrm{ViaB}$ region encoding $\mathrm{Vi}$ antigen in Salmonella typhi. J Bacteriol. 1993; $175: 4456-4465$.

21. Virlogeux I, Waxin H, Ecobichon C, Popoff MY. Role of the $v i a B$ locus in synthesis, transport, and expression of Salmonella typhi Vi antigen. Microbiology. 1995;141:3039-3047.

22. Coombes BK, Wickham ME, Lowden MJ, Brown NF, Finlay BB. Negative regulation of Salmonella pathogenicity island 2 is required for contextual control of virulence during typhoid. Proc Natl Acad Sci U S A. 2005;102:17460-17465.

23. Pickard D, Wain J, Baker S, et al. Composition, acquisition and distribution of the Vi exopolysaccharide-encoding Salmonella enterica pathogenicity island SPI-7. J Bacteriol. 2003;185: 5055-5065.

24. Zhang XL, Tsui IS, Yip CM, et al. Salmonella enterica serovar typhi uses type IVB pili to enter human intestinal epithelial cells. Infect Immun. 2000;68:3067-3073.

25. Pan Q, Zhang XL, Wu HY, et al. Identification of aptamers that preferentially bind the type IVB pili and inhibit human monocytic cell invasion by Salmonella enterica serovar typhi. Antimicrob Agents Chemother. 2005;49:4052-4060.

26. Wu HY, Zhang XL, Pan Q, Wu J. Functional selection of a type IV pili-binding peptide that specifically inhibits Salmonella Typhi adhesion to/invasion of human monocytic cells. Peptides. 2005;26:2057-2063.

27. Wang F, Zhang XL, Zhou Y, et al. Type IVB piliated Salmonella Typhi enhance IL- 6 and NF- $\kappa \mathrm{B}$ production in human monocytic THP-1 cells through activation of protein kinase C. Immunobiology. 2005;210:283-293.

28. Mirold S, Rabsch W, Rohde M, et al. Isolation of a temperate bacteriophage encoding the type III effector protein SopE from an epidemic Salmonella typhimurium strain. Proc Natl Acad Sci U S A. 1999;96:9845-9850.

29. Mirold S, Rabsch W, Tschape H, Hardt WD. Transfer of the Salmonella type III effector sopE between unrelated phage families. J Mol Biol. 2001;312:7-16.

30. Shakespeare WA, Davie D, Tonnerre C, Rubin MA, Strong M, Petti CA. Nalidixic acid-resistant Salmonella enterica serotype Typhi presenting as a primary psoas abscess: case report and review of the literature. J Clin Microbiol. 2005;43:996-998.

31. Hone DM, Attridge SR, Forrest B, et al. A galE, via (Vi antigen-negative) mutant of Salmonella typhi Ty2 retains virulence in humans. Infect Immun. 1988;56:1326-1333.

32. Arya SC. Field effectiveness of Vi polysaccharide typhoid vaccine in the People's Republic of China. J Infect Dis. 2002; 185:845; author reply 845-846.

33. Ciacci-Woolwine F, Blomfield IC, Richardson SH, Mizel SB. Salmonella flagellin induces tumor necrosis factor $\alpha$ in a human promonocytic cell line. Infect Immun. 1998;66:1127-1134.

34. Wyant TL, Tanner MK, Sztein MB. Potent immunoregulatory effects of Salmonella typhi flagella on antigenic stimulation of human peripheral blood mononuclear cells. Infect Immun. 1999;67:1338-1346.

35. Wyant TL, Tanner MK, Sztein MB. Salmonella typhi flagella are potent inducers of proinflammatory cytokine. Infect Immun. 1999;67:3619-3624.

36. Vijay-Kumar M, Wu H, Jones R, et al. Flagellin suppresses epithelial apoptosis and limits disease during enteric infection. Am J Pathol. 2006;169:1686-1700.

37. Hayashi F, Smith KD, Ozinsky A, et al. The innate immune response to bacterial flagellin is mediated by Toll-like receptor 
5. Nature. 2001;410:1099-1103.

38. Steiner TS. How flagellin and toll-like receptor 5 contribute to enteric infection. Infect Immun. 2007;75:545-552.

39. Miao EA, Alpuche-Aranda CM, Dors M, et al. Cytoplasmic flagellin activates caspase- 1 and secretion of interleukin $1 \beta$ via Ipaf. Nat Immunol. 2006;7:569-575.

40. Franchi L, Amer A, Body-Malapel M, et al. Cytosolic flagellin requires Ipaf for activation of caspase- 1 and interleukin $1 \beta$ in Salmonella-infected macrophages. Nat Immunol. 2006;7:576582.

41. Feuillet V, Medjane S, Mondor I, et al. Involvement of Toll-like receptor 5 in the recognition of flagellated bacteria. Proc Natl Acad Sci U S A. 2006;103:12487-12492.

42. Sweet MJ, Hume DA. Endotoxin signal transduction in macrophages. J Leukoc Biol. 1996;60:8-26.

43. Takeuchi O, Hoshino K, Kawai T, et al. Differential roles of TLR2 and TLR4 in recognition of gram-negative and grampositive bacterial cell wall components. Immunity. 1999;11: 443-451.

44. Mahieu T, Libert C. Should we inhibit type I interferons in sepsis? Infect Immun. 2007;75:22-29.

45. Weiss DS, Raupach B, Takeda K, Akira S, Zychlinsky A. Toll-like receptors are temporally involved in host defense. J Immunol. 2004;172:4463-4469.

46. Takeuchi O, Hoshino K, Kawai T, et al. Differential roles of TLR2 and TLR4 in recognition of gram-negative and grampositive bacterial cell wall components. Immunity. 1999;11: 443-451.

47. Bulut Y, Faure E, Thomas L, Equils O, Arditi M. Cooperation of Toll-like receptors 2 and 6 for cellular activation by soluble tuberculosis factor and Borrelia burgdorferi outer surface protein A lipoprotein: role of Toll-interacting protein and IL-1 receptor signaling molecules in Toll-like receptor 2 signaling. J Immunol. 2001;167:987-994.

48. Ozinsky A, Underhill DM, Fontenot JD, et al. The repertoire for pattern recognition of pathogens by the innate immune system is defined by cooperation between Toll-like receptors. Proc Natl Acad Sci U S A. 2000;97:13766-13771.

49. Takeuchi O, Kawai T, Muhlradt PF, et al. Discrimination of bacterial lipoproteins by Toll-like receptor 6. Int Immunol. 2001;13:933-940.

50. Hemmi H, Takeuchi O, Kawai T, et al. A Toll-like receptor recognizes bacterial DNA. Nature. 2000;408:740-745.

51. Totemeyer S, Kaiser P, Maskell DJ, Bryant CE. Sublethal infection of C57BL/6 mice with Salmonella enterica Serovar Typhimurium leads to an increase in levels of Toll-like receptor 1 (TLR1), TLR2, and TLR9 mRNA as well as a decrease in levels of TLR6 mRNA in infected organs. Infect Immun. 2005;73:1873-1878.

52. Coburn B, Grassl GA, Finlay BB. Salmonella, the host and disease: a brief review. Immunol Cell Biol. 2007;85:112-118.

53. Koebernick H, Grode L, David JR, et al. Macrophage migration inhibitory factor (MIF) plays a pivotal role in immunity against Salmonella typhimurium. Proc Natl Acad Sci U S A. 2002;99: 13681-13686.

54. Foster N, Hulme SD, Barrow PA. Induction of antimicrobial pathways during early-phase immune response to Salmonella spp. in murine macrophages: gamma interferon (IFN- $\gamma$ ) and upregulation of IFN- $\gamma$ receptor alpha expression are required for NADPH phagocytic oxidase gp91-stimulated oxidative burst and control of virulent Salmonella spp. Infect Immun. 2003;71: 4733-4741.

55. DeRoeck D, Jodar L, Clemens J. Putting typhoid vaccination on the global health agenda. N Engl J Med. 2007;357:1069-1071.
56. Boyle EC, Bishop JL, Grassl GA, Finlay BB. Salmonella: from pathogenesis to therapeutics. J Bacteriol. 2007;189:1489-1495.

57. Tacker CO, Sztein MB, Wasserman SS, et al. Phase 2 clinical trial of attenuated Salmonella enterica serovar Typhi oral live vector vaccine CVD908-htrA in US volunteers. Infect Immun. 2000;68:1196-1201.

58. Hohmann EL, Oletta CA, Killeen KP, Miller SI. phoP/ phoQ-deleted Salmonella typhi (Ty800) is a safe and immunogenic single dose typhoid fever vaccine in volunteers. J Infect Dis. 1996;173:1408-1414.

59. Mayon-White R. Re-emerging infections: Part six-exotic infections. British Journal of Infection Control. 2005;6:10-12.

60. World Health Organization. In biomedical and epidemiological research priorities of global scientific working groups. WHO Publication WHO/CDD/RES86.8 rev. 1;1987.

61. Levine MM, Herrington D, Murphy JR, et al. Safety, infectivity, immunogenicity, and in vivo stability of two attenuated auxotrophic mutant strains of Salmonella typhi, 541Ty and 543Ty, as live oral vaccines in humans. J Clin Invest. 1987;79:888-902.

62. Levine MM, Hone DM, Stocker BAD, Cadoz M. Vaccines to prevent typhoid fever. In: Woodrow G, Levine MM, eds. New Generation Vaccines. New York: Marcel Dekker, Inc; 1990: 269-287.

63. Kotton CN, Lankowski AJ, Scott N, et al. Safety and immunogenicity of attenuated Salmonella enterica serovar Typhimurium delivering an HIV-1 Gag antigen via the Salmonella Type III secretion system. Vaccine. 2006;24:62166224.

64. Rssmann H, Shams H, Poblete F, et al. Delivery of epitopes by the Salmonella Type III secretion system for vaccine development. Science. 1998;281:565-568.

65. Bumann D, Hueck C, Aebischer T, et al. Recombinant live Salmonella spp. for human vaccination against heterologous pathogens. FEMS Immunol Med Microbiol. 2000;27:357-364.

66. Pickett TE, Pasetti MF, Galen JE, et al. In vivo characterization of the murine intranasal model for assessing the immunogenicity of attenuated Salmonella enterica serovar Typhi strains as live mucosal vaccines and as live vectors. Infect Immun. 2000;68:205-213.

67. Dipetrillo MD, Tibbetts T, Kleanthous H, et al. Safety and immunogenicity of phoP/phoQ-deleted Salmonella typhi expressing Helicobacter pylori urease in adult volunteers. Vaccine. 1999;18:449-459.

68. Stokes MG, Titball RW, Neeson BN, et al. Oral Administration of a Salmonella enterica-based vaccine expressing bacillus anthracis protective antigen confers protection against Aerosolized B anthracis. Infect Immun. 2007;75:1827-1834.

69. Levine MM, Ferreccio C, Abrego P, Martin OS, Ortiz E, Cryz S. Duration of efficacy Ty21a, attenuated Salmonella typhi live oral vaccine. Vaccine. 1999; 17:S22-S27.

70. Roberts M, Chatfield SN, Dougan G. Salmonella as carriers of heterologous antigens. In: O'Hagan DT. Novel delivery systems for oral vaccines. CRC Press: Boca Raton, Florida, U S A. 1994; 27-58.

71. Curtiss R 3rd. Bacterial infectious disease control by vaccine development. J Clin Invest. 2002;110:1061-1066.

72. Luo F, Feng Y, Liu M, et al. A type IVB pili operon promoter controlling nucleocapsid gene expression of SARS-CoV in Salmonella elicits full immune response by intranasal vaccination. Clin Vaccine Immunol. 2007;14:990-997. 